\title{
The microtubule plus-end-tracking protein CLIP-170 associates with the spermatid manchette and is essential for spermatogenesis
}

\author{
Anna Akhmanova, ${ }^{1}$ Anne-Laure Mausset-Bonnefont, ${ }^{1,6}$ Wiggert van Cappellen, ${ }^{2}$ Nanda Keijzer, ${ }^{1}$ \\ Casper C. Hoogenraad, ${ }^{1,3}$ Tatiana Stepanova, ${ }^{1}$ Ksenija Drabek, ${ }^{1}$ Jacqueline van der Wees, ${ }^{1}$ \\ Mieke Mommaas, ${ }^{4}$ Jos Onderwater ${ }^{4}$ Hans van der Meulen, ${ }^{4}$ Marvin E. Tanenbaum, ${ }^{5}$ \\ Rene H. Medema, ${ }^{5}$ Jos Hoogerbrugge, ${ }^{2}$ Jan Vreeburg, ${ }^{2}$ Evert-Jan Uringa, ${ }^{2}$ J. Anton Grootegoed, ${ }^{2}$ \\ Frank Grosveld, ${ }^{1}$ and Niels Galjart ${ }^{1,}$ \\ ${ }^{1}$ Department of Cell Biology and Genetics, ${ }^{2}$ Department of Reproduction and Development, and ${ }^{3}$ Department of \\ Neuroscience, Erasmus MC, 3000 DR Rotterdam, The Netherlands; ${ }^{4}$ Center for Electron Microscopy, Department of \\ Molecular Cell Biology, Leiden University Medical Center, 2300 RA Leiden, The Netherlands; ${ }^{5}$ Department of Medical \\ Oncology, University Medical Center, 3584 CG Utrecht, The Netherlands
}

\begin{abstract}
CLIP-170 is a microtubule "plus-end-tracking protein" implicated in the control of microtubule dynamics, dynactin localization, and the linking of endosomes to microtubules. To investigate the function of mouse CLIP-170, we generated CLIP-170 knockout and GFP-CLIP-170 knock-in alleles. Residual CLIP-170 is detected in lungs and embryos of homozygous CLIP-170 knockout mice, but not in other tissues and cell types, indicating that we have generated a hypomorphic mutant. Homozygous CLIP-170 knockout mice are viable and appear normal. However, male knockout mice are subfertile and produce sperm with abnormal heads. Using the knock-in mice, we followed GFP-CLIP-170 expression and behavior in dissected, live testis tubules. We detect plus-end-tracking GFP-CLIP-170 in spermatogonia. As spermatogenesis proceeds, GFP-CLIP-170 expression increases and the fusion protein strongly marks syncytia of differentiated spermatogonia and early prophase spermatocytes. Subsequently GFP-CLIP-170 levels drop, but during spermiogenesis (post-meiotic development), GFP-CLIP-170 accumulates again and is present on spermatid manchettes and centrosomes. Bleaching studies show that, as spermatogenesis progresses, GFP-CLIP-170 converts from a mobile plus-end-tracking protein to a relatively immobile protein. We propose that CLIP-170 has a structural function in the male germline, in particular in spermatid differentiation and sperm head shaping.
\end{abstract}

[Keywords: Microtubules; plus-end-tracking proteins; CLIP-170; spermatogenesis; spermatid manchette]

Supplemental material is available at http://www.genesdev.org.

Received March 20, 2005; revised version accepted July 13, 2005.

CLIP-170 was originally identified as a microtubule (MT)-binding protein from HeLa cells (Rickard and Kreis 1990). The name "CLIP-170" (cytoplasmic linker protein of $170 \mathrm{kDa}$ ) was proposed after the discovery that this protein linked endocytic vesicles to MTs in an in vitro assay (Pierre et al. 1992). CLIP-170 was also independently identified as restin (Bilbe et al. 1992), after its increased expression level in Reed-Sternberg cells of

${ }^{6}$ Present address: INSERM U583, Institut des Neurosciences de Montpellier, Hôpital Saint-Eloi, 80 avenue Augustin Fliche, BP 74103, 34091 Montpellier Cedex 5, France.

${ }^{7}$ Corresponding author.

E-MAIL n.galjart@erasmusmc.nl; FAX 31-10-4089468.

Article and publication are at http://www.genesdev.org/cgi/doi/10.1101/ gad.344505.
Hodgkin's lymphoma. CLIP-170 consists of an N-terminal, MT-binding region, an elongated, central $\alpha$-helical domain responsible for dimerization of the protein, and C-terminal metal-binding motifs (Pierre et al. 1992). The $\mathrm{N}$ terminus, which contains two MT-binding CAP-GLY domains surrounded by basic serine-rich stretches, can interact directly with the $\mathrm{C}$ terminus of the same molecule (Lansbergen et al. 2004). This capacity of CLIP-170 to fold on itself might serve to prevent unwanted interactions. The closest mammalian relative of CLIP-170 is CLIP-115, which contains N-terminal CAP-GLY domains and a coiled-coil domain, but lacks the metalbinding motifs (De Zeeuw et al. 1997).

At low levels of overexpression, CLIP-170, when fused to GFP, was shown to associate specifically with the 
ends of growing MTs (Perez et al. 1999). This behavior, which is visualized as movement of "comet-like" dashes, has now been documented for a variety of other proteins; it has been termed "plus-end tracking," and the proteins that show it are called "+TIPs" (Schuyler and Pellman 2001). Different mechanisms account for plusend tracking (for recent review, see Akhmanova and Hoogenraad 2005). In mammalian cells, CLIP-170 displays "treadmilling"; that is, it associates with the freshly polymerized distal end of a growing MT and dissociates from the "older" MT lattice, while it remains immobile with respect to the MT (Perez et al. 1999). However, the CLIP-170 homologs in budding and fission yeast, called Biklp and Tiplp, respectively, are delivered to MT ends by kinesin motors (Busch et al. 2004; Carvalho et al. 2004).

The presence of CLIP-170 at the tips of growing MTs has focused attention toward the role of this protein in the regulation of MT dynamics. Budding yeast Bik1p has a MT-stabilizing function (Carvalho et al. 2004), and fission yeast Tip $1 \mathrm{p}$ acts as anti-catastrophe factor, preventing the premature conversion of MT growth to shrinkage in regions other than the cell ends (Brunner and Nurse 2000). Using a dominant-negative approach, it was shown that in CHO cells CLIP-115 and CLIP-170 have redundant roles as MT rescue factors, that is, they prevent MTs that have undergone catastrophe at the cell periphery from shrinking back to the centrosome (Komarova et al. 2002). This function is supported by in vitro studies, in which the dimeric head domain of CLIP-170 promotes MT rescues and induces the formation of curved oligomers of purified tubulin (Arnal et al. 2004). Together these data underscore the role of CLIPs as "positive" regulators of MT growth.

CLIP-170 interacts directly with the CLASPs, which are also +TIPs (Akhmanova et al. 2001), and with IQGAP1, a Rac1/Cdc42-binding protein (Fukata et al. 2002). These interactions may play a role in regulating MT dynamics in specific cell areas, such as the leading edge of motile fibroblasts. The Drosophila melanogaster homolog of CLIP-170, D-CLIP-190 (Lantz and Miller 1998), was shown to associate with myosin VI, indicating that D-CLIP-190 might be involved in linking the actin and MT networks. CLIP-170 is also involved in the dynein-dynactin pathway: It binds to LIS1, which is a regulator of dynein function (Coquelle et al. 2002; Tai et al. 2002). In addition, it interacts directly with the dynactin component $\mathrm{p} 150^{\text {Glued }}$ and contributes to targeting of the dynactin complex to the growing MT ends (Goodson et al. 2003; Lansbergen et al. 2004). In mitotic cells, CLIP-170 was shown to accumulate at prometaphase kinetochores, where it colocalizes with dynein, dynactin, and LIS1 (Dujardin et al. 1998; Coquelle et al. 2002; Tai et al. 2002), suggesting a function for this protein in mitosis as well as in interphase. Consistent with this distribution, a recent study using RNA interference (RNAi) revealed an essential role for CLIP-170 during mitosis in HEp-2 cells (Wieland et al. 2004).

While these studies have provided clues about cellular functions of CLIP-170, the role of this protein in the whole animal has never been addressed. In order to investigate the in vivo function of murine CLIP-170, we generated CLIP-170 knockout (KO) and GFP-CLIP-170 knock-in (KI) mice. CLIP-170 KO mice are viable, yet male KO mice produce abnormal sperm, which lack a typical, hook-shaped head morphology. We therefore focused on the role of CLIP-170 in spermatogenesis and discovered a novel, testis-specific isoform of CLIP-170. Antibody staining reveals a differentiation-stage-dependent distribution of CLIP-170 in germ cells of the testis. Using GFP-CLIP-170 KI mice, we performed live analysis in testis tubules. Dynamic, plus-end-tracking GFPCLIP-170 is detected in spermatogonia. During spermiogenesis, GFP-CLIP-170 associates with centrosomes, spermatid manchettes, and the manchette rings. Bleaching studies reveal that at these later stages, GFP-CLIP170 is mainly detected in immobile structures. Thus, CLIP-170 can act both as a +TIP and as a structural protein and is essential for normal spermatogenesis.

\section{Results}

\section{A novel exon in the CLIP-170-encoding gene}

In order to devise a gene-targeting strategy, we generated a map of the murine CLIP-170 gene (gene symbol Rsn, for restin), taking mouse cDNA clone BC007191 and human and mouse ENSEMBL genomic regions as references (Fig. 1A). The Rsn gene spans $\sim 100 \mathrm{~kb}$. Exon 1 is noncoding and is separated from the first translated exon (exon 2) by $>25 \mathrm{~kb}$. The restin-specific exon, encoding 35 amino acids (Bilbe et al. 1992), is located between exons 8 and 9 of clone BC007191, while a small "11 amino acid" exon (Griparic and Keller 1998) is located just upstream of the exon "+35" (Fig. 1A). A brain-specific splice form, represented by the rat brain cDNA AJ237670 (Akhmanova et al. 2001), lacks a part of exon 9 and the whole exon 10 (Fig. 1A). All these splice variants encode proteins with alterations within the central coiled-coil region of CLIP-170.

A short, testis-specific CLIP-170 cDNA has been described, which encodes a C-terminal isoform of CLIP170, called CLIP-50 (Tarsounas et al. 2001). The $5^{\prime}$-end of this cDNA is located upstream of exon 16, and it is collinear with CLIP-170 clone BC007191 from exon 16 to exon 24. The $5^{\prime}$-end of another mouse testis cDNA (accession no. BB616336) is located $\sim 14 \mathrm{~kb}$ downstream of exon 1 . These results suggest that in the testis, multiple transcription initiation sites exist in the CLIP-170 gene.

Surprisingly, we found a sequence of $>2 \mathrm{~kb}$ between exons 15 and 16 (Fig. 1A) that is highly conserved between man and mouse and is surrounded by consensus splice acceptor and donor sites. When translated, this domain, which has a high potential to form coiled-coils, does not interrupt the CLIP-170 coding sequence. We called this putative novel exon LORF (long open reading frame). By using RT-PCR, we confirmed that the LORF exon is abundantly expressed in the testis, but it is also present in other tissues (data not shown).

Northern blot analysis in various tissues from mouse, 
A
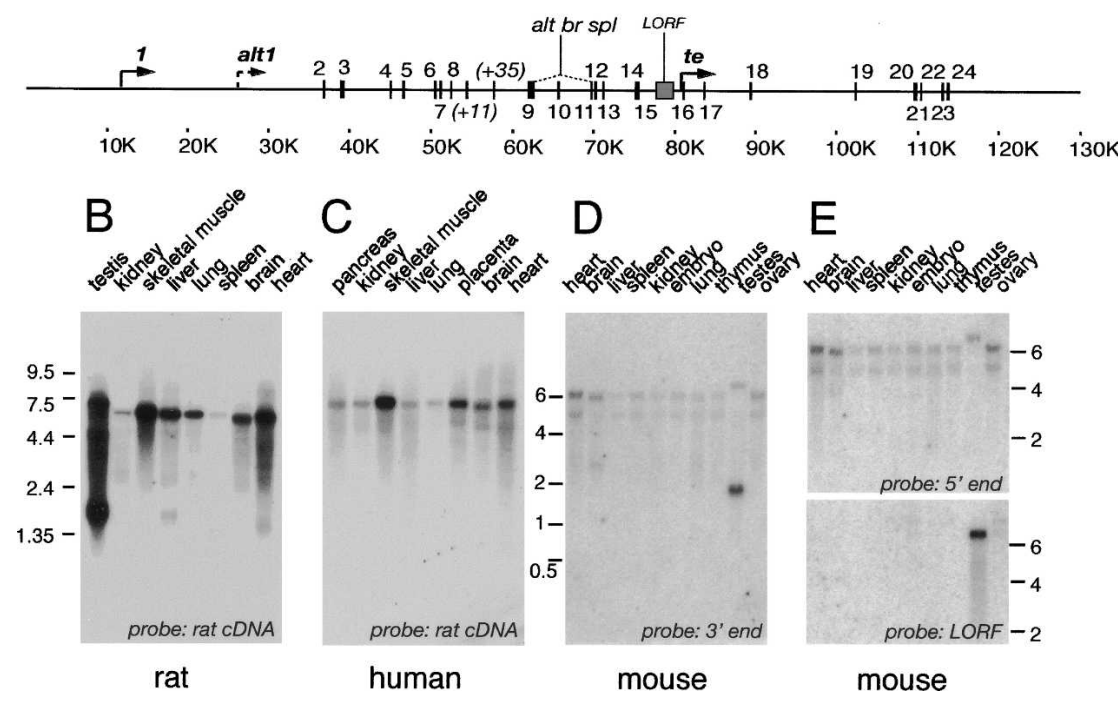

Figure 1. Genomic organization and expression of the gene encoding CLIP-170. (A) Genomic organization of the gene encoding CLIP-170. The position of common $(1-24)$ and alternatively spliced $(+11,+35$, LORF) exons and transcription initiation sites $(1$, alt 1 , te) is indicated. (Alt br spl) Alternative splice event generating the brain-specific mRNA. Scale in kilobase pairs $(\mathrm{K}) .(B-E) C L I P-170$ mRNA expression. Northern blots from rat, human, and mouse were hybridized with the indicated probes. Size markers are indicated (kilobases). rat, and human revealed ubiquitous CLIP-170 expression, with varying mRNA levels (Fig. 1B-E). In all three organisms, CLIP-170 transcripts in brain are somewhat shorter than the common mRNAs in other tissues, consistent with the smaller brain-specific splice variant (Akhmanova et al. 2001). In rat and mouse testis, multiple mRNAs are present, ranging in size from $\sim 2$ to $\sim 7$ kb (Fig. 1B,D,E). The shortest testis transcript likely corresponds to the CLIP-50 mRNA (Tarsounas et al. 2001), since it does not hybridize to the 5' part of CLIP-170 cDNA (Fig. 1E, upper panel). Hybridization of the mouse Northern blot with a LORF-specific probe revealed that the longest testis transcript contains the LORF exon (Fig. $1 \mathrm{E}$, lower panel). Taken together, these data suggest that several CLIP-170 isoforms are produced from the Rsn gene. Most notably, inclusion of the LORF exon extends the CLIP-170 coding sequence with $\sim 87 \mathrm{kDa}$.

\section{Generation of two mutant CLIP-170 alleles}

To generate a CLIP-170 KO allele, we inserted a GFPloxP-pMC1neo-loxP cassette in front of the ATG translation initiation codon in exon 2 , using homologous recombination in embryonic stem (ES) cells (Fig. 2A). In this allele, the transcription of the neomycin-resistance gene (neo) is antisense with respect to the CLIP-170 gene (Fig. 2A). To produce the GFP-CLIP-170 KI allele, we used Cre-mediated recombination at the loxP sites to remove pMClneo and fuse GFP-loxP in frame to the CLIP-170 coding sequences (Fig. 2A).

Targeting efficiency in ES cells with the GFP-loxPpMC1neo-loxP cassette and $5.5 \mathrm{~kb}$ of homologous upstream and downstream sequences was $2 \%$. Targeted ES cells were transfected with a plasmid expressing Cre recombinase, to delete pMClneo. One targeted ES cell clone, as well as one clone with correctly excised PMC1neo, were injected into blastocysts to allow germline transmission of the mutated alleles. Correct integra- tion and excision were verified by Southern blotting and PCR analysis, using upstream and downstream probes (Fig. 2B; data not shown).

We bred the modified CLIP-170 alleles back to the C57BL/6 background and generated heterozygous and homozygous CLIP-170 KO and KI mice. Northern blot analysis showed that the insertion of the GFP-loxPpMC1neo-loxP cassette results in absence of normal CLIP-170 mRNA in several KO tissues (Supplementary Fig. 1A,B; data not shown). These results were confirmed by RT-PCR (Supplementary Fig. 1C,D). Consistent with these data, we did not detect CLIP-170 by Western blot in brain lysates from homozygous CLIP-170 KO mice (Fig. 2C; Supplementary Fig. 1E).

Northern blot analysis further showed that expression of the testis-specific CLIP-50 transcript was not affected by the targeting procedure (Supplementary Fig. 1B). Western blot analysis confirmed that CLIP-50 is, indeed, expressed in testis extracts of wild-type, CLIP-170 KO, and CLIP-170 KI mice (Supplementary Fig. 1E). Thus, the targeting strategy does not perturb CLIP-50. In addition, whereas in most KO tissues, including brain, heart, skeletal muscle, mouse embryonic fibroblasts (MEFs), and testis, no CLIP-170 could be detected by Western blot (Fig. 2C-F; Supplementary Fig. 1E,F), in lung and in embryonic extracts residual CLIP-170 was observed with two different CLIP-170-specific antibodies (Fig. 2D; data not shown). We confirmed the existence of a CLIP-170 mRNA with a correctly sized 5'-end in KO lung using RT-PCR (Supplementary Fig. 1D). Therefore, insertion of the GFP-loxP-pMC1neo-loxP cassette into the CLIP170 gene does not result in ablation of protein expression in all tissues. We conclude that the CLIP-170 KO allele is a hypomorphic one.

In the GFP-CLIP-170 KI mice, a protein of $\sim 200 \mathrm{kDa}$ was produced instead of CLIP-170, which was present at similar levels compared with wild-type protein (Fig. 2C,D; Supplementary Fig. 1E,F). Equal expression levels 
Akhmanova et al.

Figure 2. Generation of CLIP-170 KO and GFP-CLIP-170 KI in alleles. (A) Targeting strategy of CLIP-170 KO and GFP-CLIP$170 \mathrm{KI}$ alleles. A segment of the murine Rsn gene is shown, with the ATG-containing exon and diagnostic restriction enzyme sites. The 5 '-end and $3{ }^{\prime}$-end homologous arms of the targeting construct are indicated by thick lines. The insertion cassette in the targeting construct is shown above the Rsn gene and contains GFP, loxP sites (arrowheads), and a neomycin resistance (neo) gene, driven by the $\mathrm{pMC1}$ promoter and containing a polyadenylation sequence $\left(A_{n}\right)$. The neo gene is transcribed antisense (see arrow) to the Rsn gene. Homologous recombination generates a CLIP-170 KO allele. Cre-mediated excision at the targeted Rsn allele leaves GFP and one loxP site. As these are inframe with CLIP-170-encoding sequences, the GFP-CLIP-170 KI allele is generated. (B) Southern blot and PCR analysis. The Southern blot demonstrates the presence of a homologously targeted CLIP-170 KO allele in ES cells. The PCR analysis with three primers allows detection of the wildtype and CLIP-170 KO or GFP-CLIP-170 KI alleles. $(C-F)$ Western blots of total protein lysates from brain $(C)$, heart, skeletal muscle, passage $30 \mathrm{MEFs}$, and lung $(D)$ or testis $(E, F)$, incubated with antibodies recognizing both CLIP-115 and CLIP-170 (\#2221), CLIP-170 only (\#2360), or the LORF-containing CLIP-170 isoform (LORF\#01), tubulin, EB3, and actin. $(C)$ The \#2221 antibodies recognize GFPCLIP-170 less well (asterisks in lanes). For each tissue, equal amounts of protein were loaded in each lane. In $F$, testis lysates were prepared from mice ranging in age from 1 wk to adult. Size markers (M) are indicated (kilodaltons).
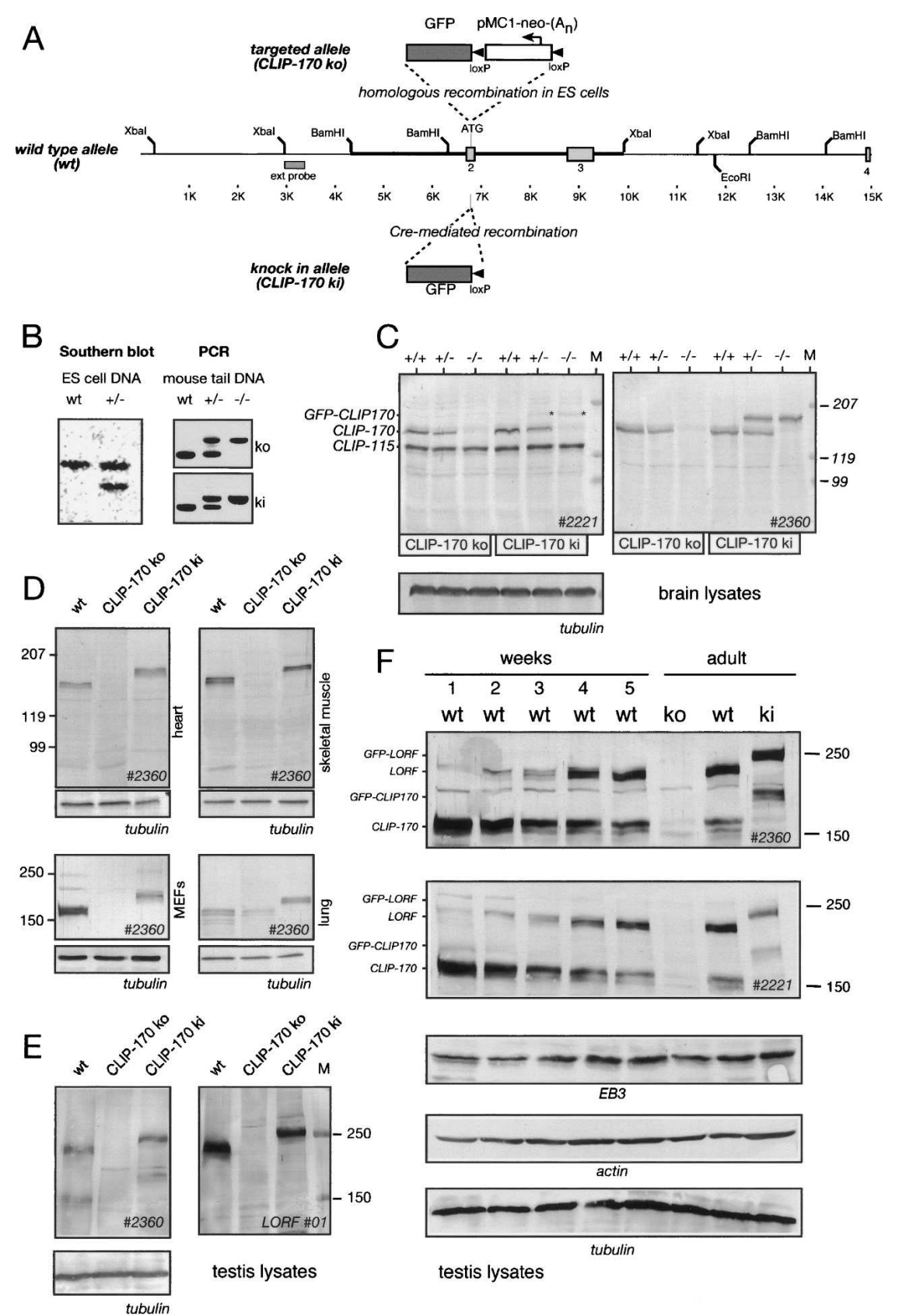

of CLIP-170 and GFP-CLIP-170 are particularly apparent in heterozygous samples. CLIP-115 levels are not altered in brain lysates from CLIP-170 KO or GFP-CLIP-170 KI mice (Fig. 2C, left panel). Thus, reduction in the level of CLIP-170 does not lead to increased CLIP-115 expression. Conversely, in CLIP-115 KO mice, CLIP-170 is not up-regulated (Hoogenraad et al. 2002).

To characterize the novel $\sim 240$-kDa LORF-containing isoform of CLIP-170, we raised rabbit polyclonal antibodies (LORF\#00 and \#01) against a bacterially produced "LORF" protein. As shown in Figure 2E, the LORF antibodies specifically recognize $\sim 240$-kDa CLIP-170 and 270-kDa GFP-CLIP-170 isoforms in testis extracts from wild-type and CLIP-170 KI mice, respectively. These

data demonstrate that the LORF exon is translated in the testis. The LORF-CLIP-170-specific signal increases upon maturation of the testis, in particular from week 3 to 4 of post-natal life (Fig. 2F). In this developmental window, the testis becomes populated with spermatids, indicating that this CLIP-170 isoform has a function in spermiogenesis rather than (pre-)meiotic stages of spermatogenesis.

\section{Analysis of CLIP-170 KO and GFP-CLIP-170 KI cell lines}

To examine the consequences of a CLIP-170 deletion at the cellular level, we derived MEFs from embryonic day 
13.5 (E13.5) embryos. We found no evidence for a severe mitotic defect in homozygous CLIP-170 KO MEFs, as compared with wild-type cells (Supplementary Fig. 2). We recently showed that depletion of CLIP-170 by RNAi reduces the ability of $\mathrm{p} 150^{\text {Glued }}$ to associate with interphase MT ends, but has no strong effect on the steadystate organelle distribution (Lansbergen et al. 2004). In line with these results, dynactin localization at MT ends is reduced in CLIP-170 KO MEFs (Fig. 3E,F), as compared with wild-type cells (Fig. 3B,C), while the level of CLIP115 at MT tips is not affected (Fig. 3A,D). The MT cytoskeleton appears normal in CLIP-170 KO cells (data not shown), perhaps because CLIP-115 is still present in these MEFs, substituting for CLIP-170. Thus, deletion of CLIP-170 in MEFs has no obvious deleterious effects, neither in interphase, nor in mitosis.

We next examined the distribution and behavior of GFP-CLIP-170 in homozygous KI MEFs. We first performed an immunoprecipitation with anti-GFP antiserum to pull down GFP-CLIP-170 and associated proteins from cultured MEFs. Results of this experiment (presented in Supplementary Fig. 1G) showed that no other GFP fusion protein is detected but full-length GFP-CLIP170 and that this protein interacts with CLASP2 and p150 Glued, as was previously shown for CLIP-170 (Akhmanova et al. 2001; Lansbergen et al. 2004). In fixed cells GFP-positive dashes are localized at the distal ends of MTs and some staining is observed in the cytoplasm (Fig. 3G-I), in line with published data (Rickard and Kreis 1990; Perez et al. 1999). The GFP-positive signals overlapped with anti-CLIP-170 antibody patterns (Supplementary Fig. 1H-J). We derived keratinocytes from homozygous CLIP-170 KO mice and transfected these with Cre recombinase to generate a mixed culture of GFPCLIP-170 KI and CLIP-170 KO cells. Only in the KI keratinocytes did we detect significant levels of p150 Glued at MT tips (Fig. 3J,K), indicating that GFP-CLIP-170 is able to direct the localization of the dynactin complex to MT plus-ends.

The behavior of GFP-CLIP-170 was further analyzed in living homozygous KI MEFs and glial cells. We observed comet-like dashes in these cell types (Fig. 3L; Supplementary Movie 1), which moved with an average velocity of $0.57 \pm 0.11 \mu \mathrm{m} / \mathrm{sec}$ in glia (60 dashes analyzed in 10 cells) or $0.50 \pm 0.08 \mu \mathrm{m} / \mathrm{sec}$ in MEFs (25 dashes analyzed in three cells; in both cases the standard deviation is indicated). This velocity is similar to the values obtained by us in cells overexpressing low levels of various +TIPs (Akhmanova et al. 2001; Stepanova et al. 2003). Treatment of cells with low doses of nocodazole and taxol immediately abolished the dynamic dashes in GFPCLIP-170 KI cells (data not shown). Taken together, these data suggest that GFP-CLIP-170, expressed from the modified Rsn allele, recapitulates the behavior and functionality of CLIP-170 in cultured cells and can be used as a tool to analyze the distribution and dynamics of CLIP-170 in vivo. This conclusion is in line with previous observations, that an overexpressed GFP-CLIP-170 fusion protein is functional in cultured cells (Perez et al. 1999; Komarova et al. 2002; Stepanova et al. 2003).
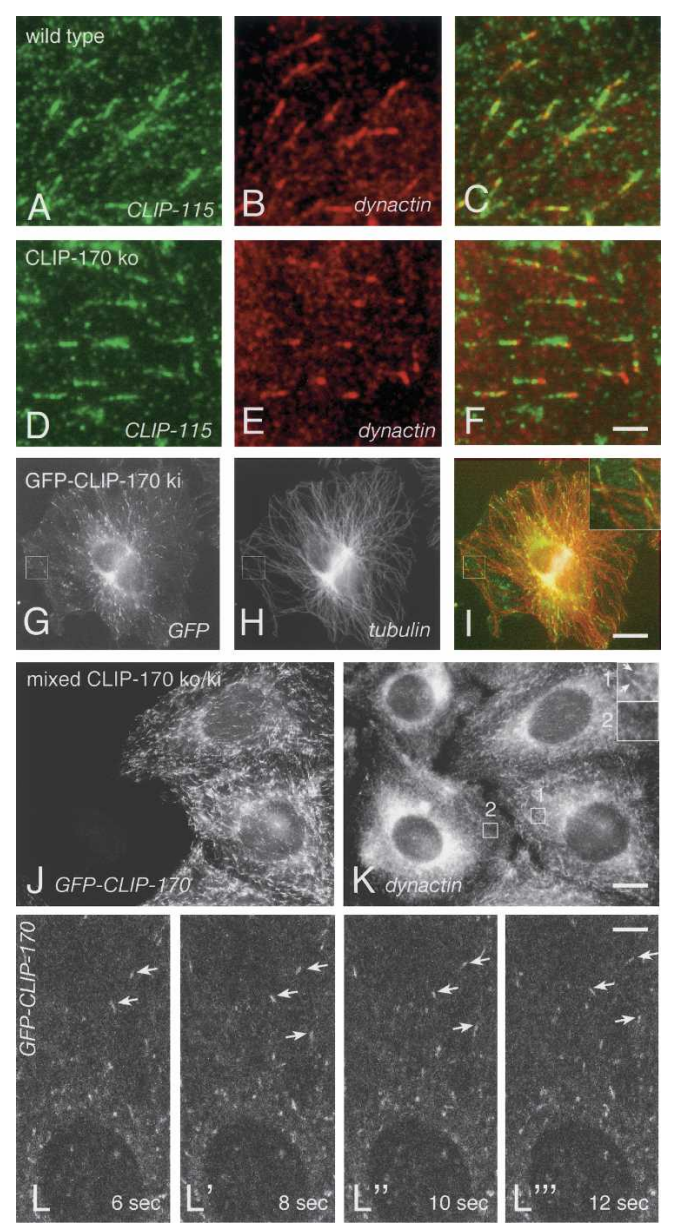

Figure 3. Cellular analysis of the CLIP-170 deletion and GFPCLIP-170 insertion. $(A-F)$ CLIP-115 and dynactin distribution in wild-type $(A-C)$ and CLIP-170 KO MEFs $(D-F)$. CLIP-115 (green) and dynactin (red) were detected with antibodies \#2238 and antibodies against dynamitin, respectively. Both CLIP-115 and dynactin localize at the ends of MTs, but dynactin signal is diminished in KO MEFs. Bar, $2.5 \mu \mathrm{m}$. $(G-I)$ GFP-CLIP-170 distribution in KI MEFs. Direct GFP fluorescence (green) was observed in combination with anti-tubulin staining (red). Enlarged MT plus-ends are shown in the inset. Bar, $10 \mu \mathrm{m} .(J, K)$ Dynactin distribution in keratinocytes. A mixed culture of CLIP-170 KO and GFP-CLIP-170 KI keratinocytes was made by transfecting a KO culture with Cre recombinase. GFP-CLIP-170 is shown in $J$ (direct fluorescence) and dynactin in $K$. Notice the decreased dynactin staining at MT ends in KO cells (see also insets in $K)$. Bar, $13 \mu \mathrm{m}$. $\left(L-L^{\prime \prime \prime}\right)$ Behavior of GFP-CLIP-170 in cultured glial cells, derived from the GFP-CLIP-170 KI mice, analyzed by time-lapse imaging. The movie of this analysis is shown as Supplementary Movie 1. $L-L^{\prime \prime \prime}$ represent parts of the images acquired after $6,8,10$, and $12 \mathrm{sec}$. The arrows indicate three examples of moving GFP-CLIP-170 dashes. Bar, $3 \mu \mathrm{m}$.

\section{Deletion of CLIP-170 affects spermatogenesis}

Heterozygous and homozygous CLIP-170 KO and GFPCLIP-170 KI mice are born in Mendelian ratios, have normal weights, and show no gross abnormalities /data not shown). Therefore, modification of the Rsn allele does not have a significant negative influence on mouse 
development. However, homozygous CLIP-170 KO male mice produce virtually no offspring. This points to a defect in male germ cell development and/or functioning, and we therefore investigated the testes of these mice.

Comparison of hematoxylin/eosin (H/E)-stained testis sections of CLIP-170 KO, KI, and wild-type mice showed no obvious morphological abnormalities in the size or organization of the testis tubules or the interstitium (Fig. 4A-C). Detailed examination of these sections, however, indicated that $\mathrm{KO}$ mice produce abnormal sperm. Analysis of sperm from the epididymis demonstrated that the CLIP-170 KO mice produce normal sperm numbers and that spermatozoa are motile, but that all sperm have an abnormal morphology; 100\% display severe head abnormalities, whereas $20 \%$ of these show combined head and tail defects (Table 1, two mice were analyzed per genotype; see also Fig. 4D-F). The main characteristic of the abnormal head is a lack of the typical hook-shaped appearance (Fig. 4E), suggesting a defect in nuclear shaping
(Russell et al. 1991). This may explain the fertility problems of male homozygous CLIP-170 KO mice. The sperm morphology was completely normal in GFP-CLIP$170 \mathrm{KI}$ animals (Fig. 4F), indicating that GFP-CLIP-170 can substitute for the function of endogenous CLIP-170 in vivo.

\section{Localization of CLIP-170 in the testis}

As shown in Figure 2E and F, deletion of CLIP-170 has no effect on the total testis levels of the major cytoskeletal proteins actin and tubulin, or EB3, another +TIP. To understand the molecular mechanism underlying the abnormal sperm phenotype in CLIP-170 KO mice, we investigated CLIP-170 localization in the testis. Using two different antibodies against CLIP-170 (\#2221 and \#2360), we detected abundant staining in spermatids, starting from step 8, as well as a weaker signal in subsets of spermatogonia of wild-type testis (Fig. 4G-J). No CLIP-

Figure 4. Testicular analysis of the CLIP-170 deletion and GFP-CLIP-170 insertion. $(A-C) \mathrm{H} / \mathrm{E}$-stained 7- $\mu \mathrm{m}$ sections of the testes from wild-type, CLIP$170 \mathrm{KO}$, and GFP-CLIP-170 KI mice. Bar, $50 \mu \mathrm{m}$. $(D-F)$ Sperm preparations, isolated from the epididymis of wild-type, CLIP-170 KO, and GFP-CLIP-170 KI mice. Bar, $10 \mu \mathrm{m} .(G-R)$ Immunofluorescence analysis. Wild-type $(G-J)$, CLIP-170 KO $(K-N)$, and GFP-CLIP-170 KI $(O-R)$ paraffin-embedded sections were incubated with the indicated antibodies and counterstained with DAPI to visualize nuclei. In the overlay in $Q$, anti-GFP staining is shown in green, and LORF\#00 in red. Bar, $40 \mu \mathrm{m}$.
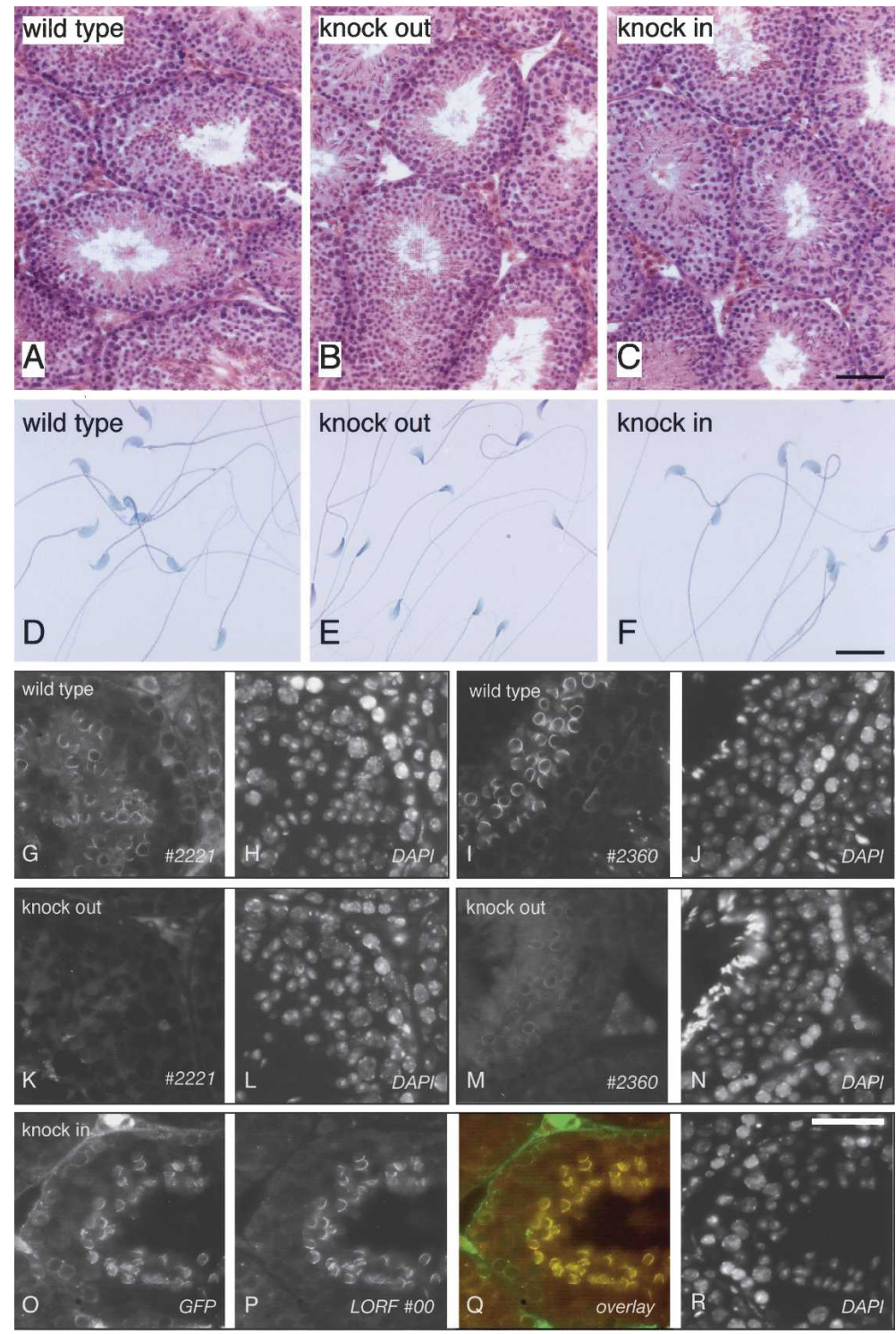
Table 1. Reproductive organ weights and sperm counts of wild-type, CLIP-170 KO, and GFP-CLIP-170 KI mice

\begin{tabular}{|c|c|c|c|c|c|c|c|}
\hline \multirow[b]{2}{*}{ Genotype } & \multicolumn{4}{|c|}{ Weight } & \multicolumn{3}{|c|}{ Sperm } \\
\hline & $\begin{array}{l}\text { Body } \\
\text { (g) }\end{array}$ & $\begin{array}{l}\text { Testis } \\
(\mathrm{mg})^{\mathrm{a}}\end{array}$ & $\begin{array}{l}\text { Epididymis } \\
(\mathrm{mg})^{\mathrm{a}}\end{array}$ & $\begin{array}{c}\text { Seminal } \\
\text { vesicle }(\mathrm{mg})^{\mathrm{a}}\end{array}$ & $\begin{array}{l}\text { Count per } \\
\text { epididymis }{ }^{\mathrm{b}}\end{array}$ & $\begin{array}{l}\text { Abnormal } \\
\text { morphology }\end{array}$ & Motility \\
\hline \multirow[t]{2}{*}{ Wild type } & 25.1 & 173 & 59 & 56 & 19 & $15 \%(7 \mathrm{H} ; 6 \mathrm{~T} ; 2 \mathrm{HT})^{\mathrm{c}}$ & $75 \%$ \\
\hline & 22.7 & 171 & 50 & 40 & 21 & $20 \%(5 \mathrm{H} ; 14 \mathrm{~T} ; 1 \mathrm{HT})$ & $68 \%$ \\
\hline \multirow[t]{2}{*}{ Knockout } & 29.8 & 194 & 71 & 62 & 18 & $100 \%(81 \mathrm{H} ; 19 \mathrm{HT})$ & $63 \%$ \\
\hline & 20.8 & 157 & 49 & 39 & 18 & $100 \%(80 ; 20 \mathrm{HT})$ & $65 \%$ \\
\hline \multirow[t]{2}{*}{ Knock-in } & 30.5 & 217 & 83 & 76 & 36 & $16 \%(7 \mathrm{H} ; 7 \mathrm{~T} ; 2 \mathrm{HT})$ & $64 \%$ \\
\hline & 20.2 & 157 & 42 & 33 & 12 & $13 \%(7 \mathrm{H} ; 5 \mathrm{~T} ; 1 \mathrm{HT})$ & $71 \%$ \\
\hline
\end{tabular}

${ }^{\mathrm{a} C}$ Combined weight of left and right tissue.

${ }^{\mathrm{b}}$ Only right tissue counted, $\times 10^{6}$.

${ }^{\mathrm{c}}(\mathrm{H})$ Percent head abnormality; $(\mathrm{T})$ percent tail abnormality; $(\mathrm{HT})$ percent combined head and tail abnormality.

170 staining was detected in Sertoli cells. In CLIP-170 KO sections, we observed weak staining with \#2221 antibodies (which recognize the $\mathrm{N}$ termini of CLIP-170 and CLIP-115) in the interstitium and in the lumen of the tubule, but not in germ cells (Fig. 4K). In contrast, staining with the \#2360 antiserum (which recognizes the C terminus of CLIP-170) revealed a weak staining in spermatids of the KO mice (Fig. 4M). Consistent with Northern and Western blot results (Supplementary Fig. 1), these data suggest that CLIP-50 (which can be recognized by \#2360, but not by \#2221) is produced in CLIP-170 KO mice and associates with spermatids. DAPI images showed that the arrangement of the cells in the testis tubule appears normal despite the absence of CLIP-170 (Fig. 4L,N).

Anti-LORF antibodies produced no specific staining in CLIP-170 KO sections (data not shown), while they reacted strongly with the elongating spermatids of the wild-type and GFP-CLIP-170 KI mice (Fig. 4O-R; data not shown). However, these antibodies did not stain spermatogonia well, which was particularly obvious when anti-GFP and anti-LORF signals were compared in the same sections of GFP-CLIP-170 KI testes (Fig. 4O-Q). Thus, the longest isoform of CLIP-170 preferentially localizes to post-meiotic germ cells, consistent with the Western blot results of Figure 2F. Taken together, these data suggest that CLIP-170 is an important component of male germ cells, with a prominent localization in spermatids.

\section{CLIP-170 is involved in spermatid manchette formation}

Cryosections from GFP-CLIP-170 KI testis showed a GFP signal in spermatogonia and brighter staining as dots and ring-like structures in elongating spermatids (Fig. 5A,B). GFP-CLIP-170 dots colocalize with or are adjacent to $\gamma$-tubulin-positive structures (Fig. 5C-E), indicating that CLIP-170 associates with one of the centrioles. The ring-like GFP-CLIP-170 signals colocalize with tubulin (data not shown), dynactin (Fig. 5F-H), and EB3 (Fig. 5I-K). They correspond to spermatid manchettesparallel arrays of MTs that form a container around the elongating spermatid nucleus (Wolosewick and Bryan
1977; Russell et al. 1991; Kierszenbaum 2001). Manchette MTs emanate from circular structures, called the manchette rings. GFP-CLIP-170 signals are particularly prominent at the manchette rings and are also present along the MTs. Manchette localization of GFP-CLIP-170 in KI mice is consistent with the CLIP-170 antibodystaining patterns in wild-type animals (Figs. 4G,I,O, 5L$\mathrm{N}, \mathrm{Q})$. High-resolution imaging with the anti-LORF antisera also revealed specific association with the spermatid manchette (Fig. 5O,P; see also Supplementary Movie 2, which shows a $3 \mathrm{D}$ representation).

In normal mice, the manchette begins to form in step 8 spermatids, when the shape of the nucleus changes from spherical to slightly elongated. In step 9 the nucleus becomes even more elongated, and the manchette runs parallel to the nuclear envelope. Observations by fluorescent microscopy revealed that in CLIP$170 \mathrm{KO}$ mice, developing manchettes with EB3-positive rings are present in step 8-9 spermatids (Fig. 6A-F). At later stages (steps 11-13), when manchettes undergo a transition from a relatively symmetric cylindrical MT array to an asymmetric configuration (Fig. 5Q), striking abnormalities were observed in the distribution of tubulin, EB3 (Fig. 6G-I; see also Supplementary Movie 3, which shows a 3D representation), and dynactin (Fig. $6 \mathrm{~J}, \mathrm{~K})$, which were often present only on one side of the nucleus. This correlated with defects in the morphology of the nuclei that failed to acquire their normal slender shape with a curved rostral end, but instead had an irregular, often club-like appearance (Fig. 6H,K).

Electron microscopy (EM) analysis showed that in wild-type step 8 spermatids, the onset of manchette formation was clearly demarcated by a narrow belt of MTs that covered approximately half of the nucleus, with the acrosome spanning the other half (Fig. 7A). In CLIP-170 KO mice, abnormal manchette features could be observed already at this stage: The belt of MTs did not span the nucleus properly, and MTs were present in aberrant areas such as within the acrosome and the nucleus (Fig. 7B). Steps 9-13 in KO mice presented absent or greatly reduced manchette MTs along the nuclear envelope, ectopic MTs along regions of the nucleus that normally do not display manchettes, or MTs at one side of the nucleus only (Supplementary Fig. 3A; data not shown). 

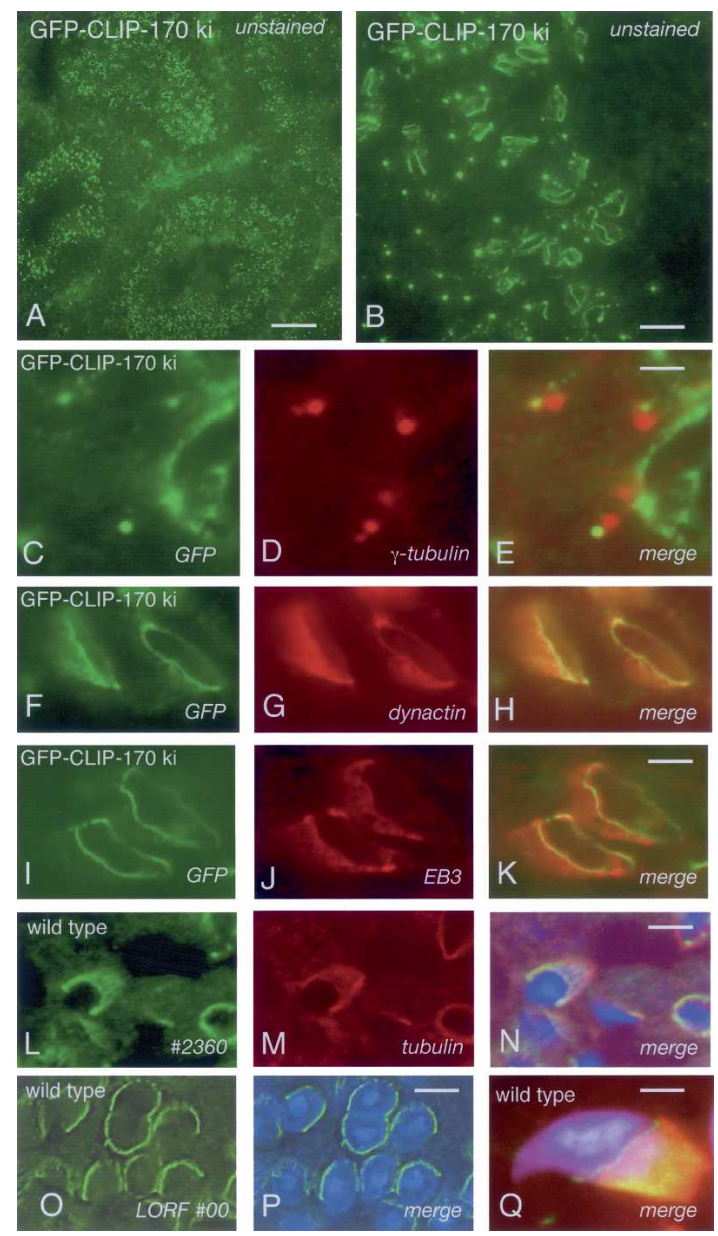

Figure 5. CLIP-170 and GFP-CLIP-170 distribution in wild-type and $\mathrm{KI}$ testis. $(A, B)$ Unstained cryosections from GFP-CLIP-170 KI mice. Bars: $A, 40 \mu \mathrm{m} ; B, 8 \mu \mathrm{m}$. $(C-K)$ Localization of GFPCLIP-170 to the centrosomes and the spermatid manchette. Testis cryosections from GFP-CLIP-170 KI mice were incubated with antibodies against $\gamma$-tubulin $(C-E)$, dynactin subunit $\mathrm{p} 150^{\text {Glued }}$ $(F-H)$, or EB3 $(I-K)$. GFP was detected by its direct fluorescence. Bars: $E, 1.5 \mu \mathrm{m} ; K, 2.5 \mu \mathrm{m}$. (L-Q) Paraffin-embedded testis sections from wild-type mice were incubated with \#2360, or LORF antibodies (green), $\beta$-tubulin antisera (red), and counterstained with DAPI (blue). In $L-N$, images were acquired with a confocal microscope and deconvolved. Notice how CLIP-170 colocalizes with tubulin. In $O$ and $P$, images were acquired with an epifluorescent microscope and deconvolved. A $3 \mathrm{D}$ representation of the LORF-CLIP-170 distribution is shown in Supplementary Movie 2. In $Q$, the image was acquired with an epifluorescent microscope and is only shown merged. Bars: $N, P, 5 \mu \mathrm{m} ; \mathrm{Q}, 1.4 \mu \mathrm{m}$.

In addition, manchette MTs from KO mice were frequently localized in membrane-bound regions that seemed to lie within spermatid nuclei (see arrows in Fig. 7B,D; Supplementary Fig. 3B). From step 11 onward, MTs were organized in a regular fashion in elongating spermatids from wild-type and GFP-CLIP-170 KI mice (Fig. 7C,E; Supplementary Fig. 3C), but they were severely disorganized in sections from $\mathrm{KO}$ mice (Fig. 7D,F). This disturbed manchette formation seemed to influence the nuclear shaping, resulting in a more rounded nucleus than in wild-type animals (Fig. 7, cf. C and D; Supplementary Fig. 3B). In addition, MTs in CLIP-170 $\mathrm{KO}$ mice were often aggregated in large cytoplasmic clusters (Supplementary Fig. 3D). Finally, in step 16 spermatids, the final stage before sperm release from Sertoli cells, the nuclei in the CLIP-170 KO testis were highly irregular in shape (Supplementary Fig. 3F).

Previous EM studies demonstrated that manchette MTs are connected by cross-bridges (Wolosewick and Bryan 1977; see also Fig. 7E; Supplementary Fig. 3C). We measured the distance between MTs at sites of crossbridges in transverse sections from wild-type, $\mathrm{KO}$, and $\mathrm{KI}$ mice (Table 2). We restricted our analysis to regions where regularly spaced MTs could be detected. Since MT organization is severely affected in CLIP-170 KO spermatids (Fig. 7), such sites are rarely seen, compared with wild-type or GFP-CLIP-170 KI sections. The data in Table 2 show that average cross-bridge size is very similar in wild-type and CLIP-170 KO spermatids; this distance correlates well with previous results (Wolosewick and Bryan 1977). Surprisingly, cross-bridges in GFPCLIP-170 KI spermatids are slightly smaller, the size dif-
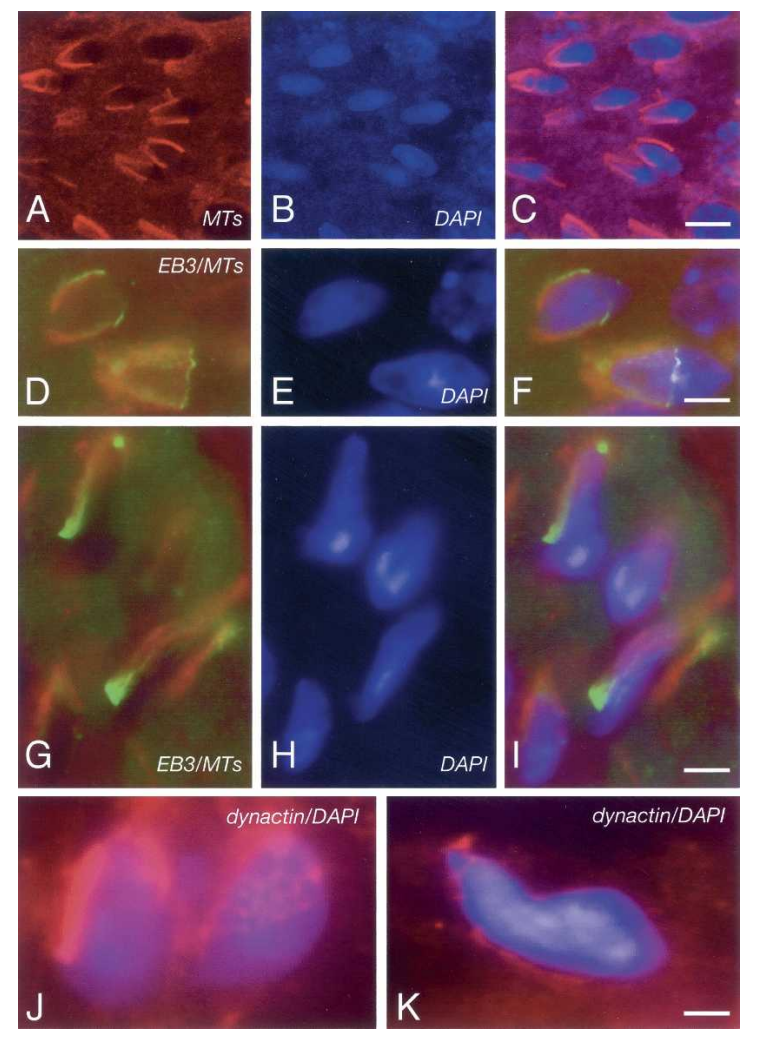

Figure 6. Immunofluorescent analysis of CLIP-170 KO testis. $(A-I)$ Paraffin-embedded testis sections from CLIP-170 KO mice were incubated with antibodies against $\beta$-tubulin $($ red $)(A-C)$, or $\beta$-tubulin (red) and EB3 (green) $(D-I)$ and counterstained with DAPI (blue). A 3D representation of EB3 and MT distribution is shown in Supplementary Movie 3. Bars: $C, 8 \mu \mathrm{m}_{;} F, 2.5 \mu \mathrm{m} ; I_{1} 4$ $\mu \mathrm{m} .(J, K)$ Cryosections of the testis from CLIP-170 KO mice were incubated with antibodies against dynactin subunit p150 ${ }^{\text {Glued }}$ (red) and counterstained with DAPI (blue). Bar, $1 \mu \mathrm{m}$. 
ference being highly significant $(P<0.005$; ANOVA, single-factor analysis). We also counted more cross-bridges per MT in cross-sections in the GFP-CLIP-170 KI spermatids, than in wild-type and KO mice (Table 2). Together, these results indicate that CLIP-170 is not an essential cross-bridge component; however, given the disorganized manchettes in the CLIP-170 KO and the smaller size and increased number of cross-bridges in GFP-CLIP-170 KI mice, we propose that CLIP-170 influences the formation of cross-bridges between manchette MTs.
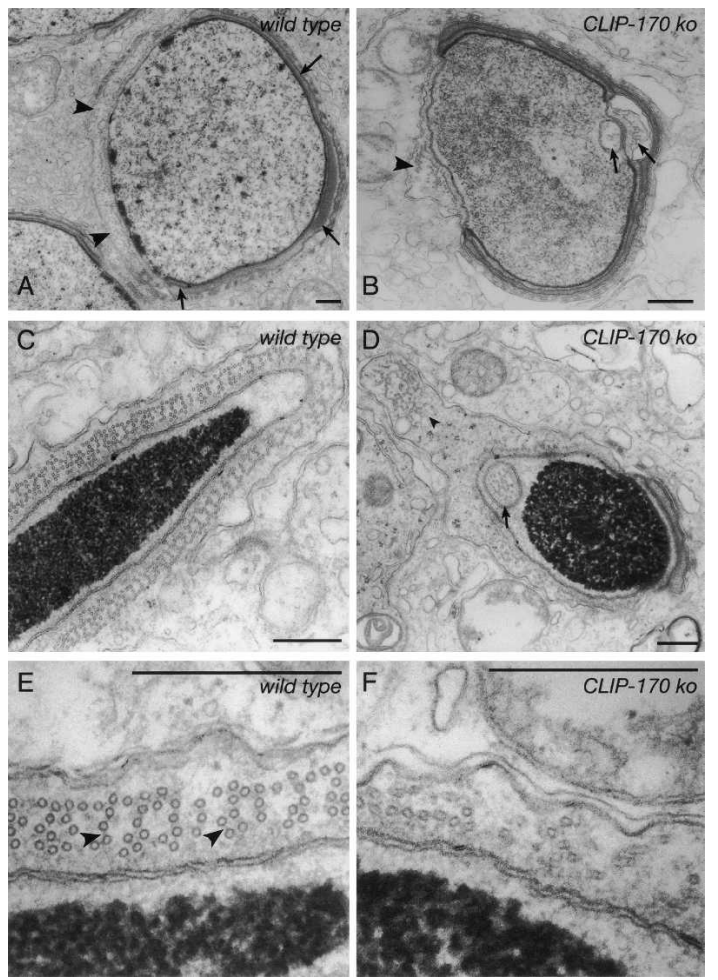

Figure 7. Ultrastructural morphology of the testis of wild-type and CLIP-170 KO mice. (A) Step 8 spermatid from a wild-type mouse with manchette MTs (indicated by arrowheads) located in close apposition to the nucleus in the area not occupied by the acrosome (indicated by arrows). (B) Step 8 spermatid from a CLIP-170 KO mouse. Some manchette MTs appear to be properly positioned (arrowhead). However, they do not completely cover the area of the nucleus that is free of the acrosome. Moreover, bundles of MTs show ectopic expression and are positioned within the nucleus (arrows). $(C, D)$ Transverse sections from wild-type $(C)$ or CLIP-170 KO $(D)$ step 14 spermatids, showing portions of the manchette and nucleus. In the wildtype spermatid, the nucleus is surrounded by highly regularly organized MT bundles, which form a well-developed manchette. The CLIP-170 KO nucleus is almost round, and the manchette shows ectopic expression with MTs detached from the nucleus (arrowhead) and within the nucleus (arrow). (E) Higher magnification of a part of the wild-type manchette shown in $C$. The cross-sectioned microtubules can be seen arranged in a regular pattern, with prominent cross-bridges (arrowheads). (F) High magnification of a part of the KO manchette shown in $D$. Cross-sectioned MTs in KO mice display a highly irregular arrangement. Not all MTs are truly cross-sectioned, indicating that they do not run in parallel arrays. Bars, $0.5 \mu \mathrm{m}$.
Table 2. Size and number of MT cross-bridges in spermatid manchettes

\begin{tabular}{lcc}
\hline Genotype & $\begin{array}{c}\text { Cross-bridge size } \\
(\mathrm{nm} \pm \mathrm{SD})\end{array}$ & $\begin{array}{c}\text { Cross-bridges } \\
\text { MTs }(\%)\end{array}$ \\
\hline Wild type & $13.46 \pm 4.48(n=107)$ & $106 / 907(11.6)$ \\
CLIP-170 knockout & $13.38 \pm 4.37(n=94)$ & $97 / 603(16)$ \\
GFP-CLIP-170 knock-in & $11.64 \pm 3.57(n=131)$ & $165 / 662(25)$ \\
\hline
\end{tabular}

(SD) Standard deviation.

\section{GFP-CLIP-170 distribution in live testis tubules}

To visualize GFP-CLIP-170 in live germ cells, we dissected testis tubules (with or without Hoechst) and examined these at $33^{\circ} \mathrm{C}$ with a confocal/multiphoton setup. Propidium iodide staining revealed that hardly any cells died using this procedure (data not shown). Moreover, sperm tails kept beating for several hours, and we could observe dynamic GFP-CLIP-170 (see below). Thus, this procedure appears suitable to follow the behavior of GFP-CLIP-170 in testis tubules.

The distribution of GFP-CLIP-170 was examined by scanning tubules longitudinally and acquiring optical sections through the tubule. Bright signals were observed in the cytoplasm of cells that formed syncytia close to the basal lamina. The number of positive cells within a syncytium differed (e.g., Fig. 8, cf. A and B). The GFP-CLIP-170-positive cells in Figure 8A and B (which are still images of Supplementary Movies 4, 5) have oval nuclei with a diameter of $\sim 7 \mu \mathrm{m}$ (18 nuclei counted). In combination with Hoechst nuclear staining and numbers of cells in syncytia, we identified these cells as type B spermatogonia and pre-leptotene primary spermatocytes (Vigodner et al. 2002). In addition, we detected strong GFP signals closer to the lumen of the tubule, in elongating spermatids (Fig. 8C,D; Supplementary Movies 6,7$)$. We did not observe any GFP-CLIP-170 in Sertoli cells.

Cells that expressed somewhat lower levels of GFPCLIP-170 were located very near to the basal lamina. Nuclear sizes in these cells ranged from $9 \times 10 \mu \mathrm{m}$ to $12 \times 15 \mu \mathrm{m}$. Based on these observations and Hoechst staining, these cells were identified as undifferentiated (e.g., Fig. 8F) and differentiated (e.g., Fig. 8E) type A spermatogonia (see also Supplementary Movies 8, 9). Starting at undifferentiated A-paired spermatogonia (Fig. 8F), the male germline cells form syncytia, connected by intercellular cytoplasmic bridges. GFP-CLIP-170 nicely visualizes these bridges.

All signals were specific and not due to autofluorescence, since imaging of wild-type testis tubules with the same settings revealed no green fluorescent signal (data not shown). Thus, GFP-CLIP-170 is expressed in a highly specific pattern in germ cells at different steps of spermatogenesis.

\section{Dynamic behavior of GFP-CLIP-170 in the testis}

To examine the dynamics of GFP-CLIP-170 movement in the testis, we performed time-lapse imaging studies 
Figure 8. Behavior of GFP-CLIP-170 in live testis tubules. Testis tubules were dissected from GFPCLIP-170 KI mice (in some cases treated with Hoechst) and analyzed with a confocal/multiphoton microscope. (A) Low-magnification view of GFPCLIP-170 distribution. The panel shows an image from Supplementary Movie 4. Notice the accumulation of GFP-CLIP-170 in pre-leptotene spermatocytes. Bar, $30 \mu \mathrm{m}$. (B) Low-magnification view of GFP-CLIP-170 and Hoechst distribution. The panel shows an image from Supplementary Movie 5. Notice the accumulation of GFP-CLIP-170 in type B spermatogonia and the absence of signal in Sertoli cells. Bar, $25 \mu \mathrm{m} .(C)$ High-magnification view of GFP-CLIP-170 distribution in elongating spermatids. Analysis was as in $A$. Notice the accumulation of GFP-CLIP-170 on manchettes, the manchette ring, and on centrosomes. Bar, $5 \mu \mathrm{m}$. (D) High-magnification view of GFP-CLIP-170 and Hoechst distribution in elongating spermatids. Analysis was as in $B$. This panel shows one plane from Supplementary Movie 7. Notice the accumulation of GFP-CLIP-170 in elongating but not in the round spermatids. Bar, 6 $\mu \mathrm{m}$. $\left(E-E^{\prime \prime \prime \prime \prime}\right)$ Time-lapse analysis of GFP-CLIP-170. Analysis was as in $B$. In $E$, a combined GFP and Hoechst image is shown to identify the germ cell as a differentiated type A spermatogonium. Subsequent time-lapse analysis was done with the 488-nm laser only and is shown in Supplementary Movie 8. $E^{\prime}-E^{\prime \prime \prime \prime \prime}$ show part of the images (see rectangle in $E$ ) acquired after $3,6,9,12$, and $15 \mathrm{sec}$, respectively. Arrows indicate GFP-CLIP-170-positive dashes. Bar, $6 \mu \mathrm{m}$. $(F-I)$ FRAP/FLIP analysis of GFP-CLIP-170 behavior. Live images of a portion of a testis tubule were taken, and after a number of frames, the rectangular regions indicated with the number 1 were bleached and time-lapse analysis was resumed. In $F$ and $H$, undifferentiated spermatogonia (A-paired) were analyzed, whereas in $G$ and $I$, elongating spermatids were analyzed. $H$ and $I$ show the relative fluorescent intensity (percentage) of the indicated regions of interest (ROIs) during the experiment. Whereas GFP-CLIP-170 is largely mobile in spermatogonia, it is largely immobile in spermatids. Bars: $F, 9 \mu \mathrm{m} ; G, 6 \mu \mathrm{m}$.
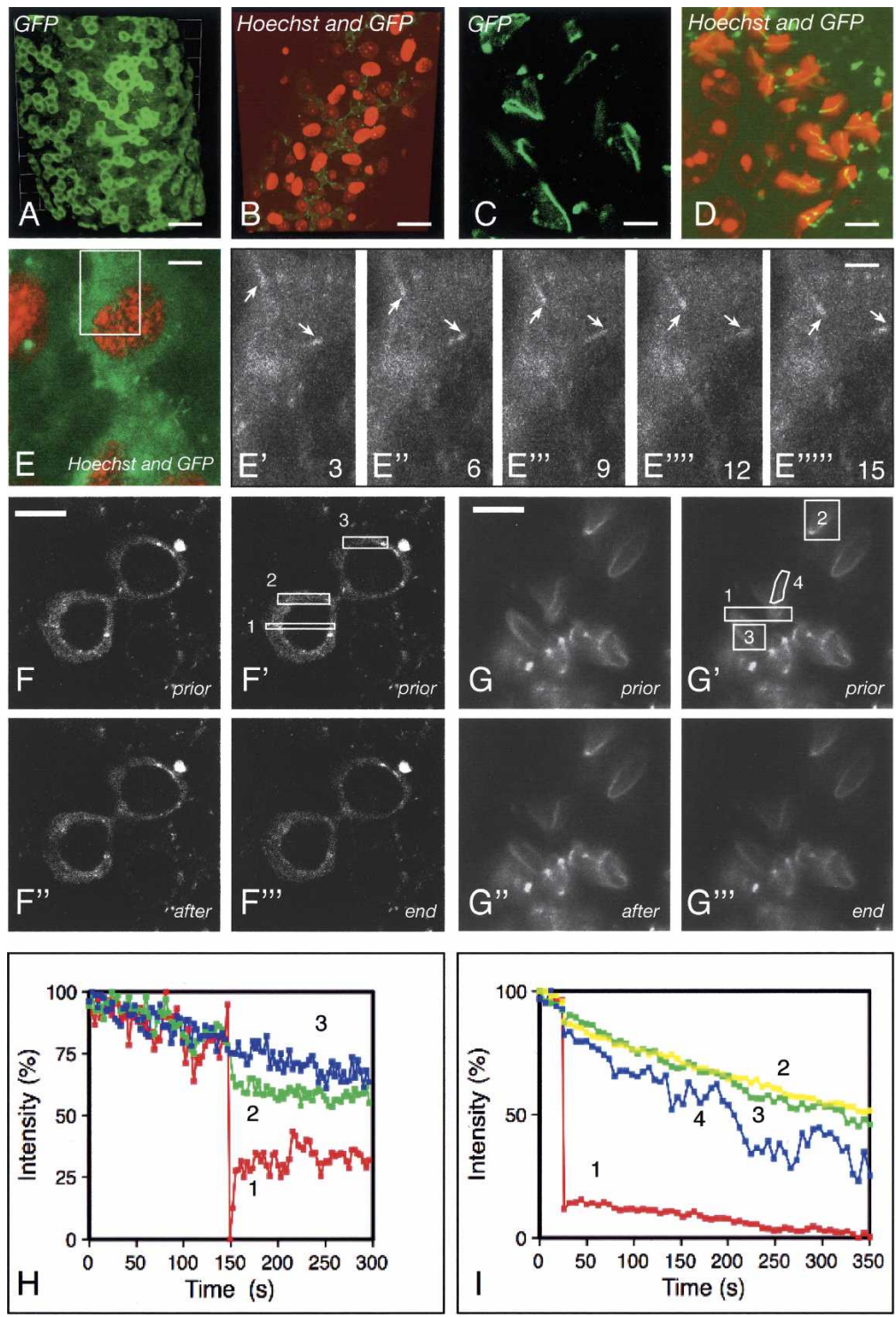

on the types of cells described above. Only in cells near the basal lamina, which express relatively low levels of GFP-CLIP-170, did we detect comet-like dashes, typical of GFP-CLIP-170 in cultured cells (Fig. 8E; Supplementary Movies $8,9 \mid$. The speed of movement of GFP-positive dashes was $0.16 \pm 0.04 \mu \mathrm{m} / \mathrm{sec}$ (55 dashes counted in 12 cells), which is lower than the speed observed in cultured glia or MEFs, because of lower observation temperature (Akhmanova et al. 2001; Stepanova et al. 2003). In a few cells, we observed both moving dashes and immobile signal (Fig. 8F; Supplementary Movie 9). However, in the majority of germ cells in the testis, we did not detect any movement of GFP-CLIP-170.

We used fluorescence recovery after photobleaching (FRAP) and fluorescence loss in photobleaching (FLIP) to further examine the dynamic properties of GFP-CLIP170. In cells with predominantly plus-end-tracking GFP-
CLIP-170, a fast recovery of fluorescence in the bleached area was observed, which was complete after $\sim 1 \mathrm{~min}$ (Fig. $8 \mathrm{~F}, \mathrm{H}$; Supplementary Fig. 4A,B). Fluorescence recovery was accompanied by fluorescence loss in nonbleached areas of the same cell. When cells with dynamic GFPCLIP-170 were completely bleached, very slow fluorescence recovery was observed (data not shown), indicating that GFP-CLIP-170 can pass through intercellular bridges from one cell of the syncytium to another.

In pre-leptotene spermatocytes with high GFP-CLIP170 signal, FRAP-FLIP analysis revealed recovery of fluorescent signal in the bleached area in $\sim 5 \mathrm{~min}$, which was accompanied by loss in fluorescent signal in nonbleached regions of the same cell (data not shown). In contrast, analysis in elongating spermatids showed relatively immobile GFP-CLIP-170 during the course of the experiment (Fig. 8G,I; Supplementary Fig. 4C-F; Supple- 
mentary Movies 10, 11). These data indicate that GFPCLIP-170 becomes immobile as male germ cells differentiate.

\section{Discussion}

CLIP-170 is the founding member of the +TIP group of MT-associated factors (Perez et al. 1999). Since nothing is known about the in vivo function of mammalian CLIP-170, we wished to address this using gene-targeting technology. The documentation of dominant-negative properties of a C-terminal CLIP-170 mutant (Komarova et al. 2002) and the finding of CLIP-50 (Tarsounas et al. 2001) suggest that complete inactivation of CLIP-170 function can only be achieved by deleting the whole gene. However, since the Rsn gene spans $\sim 100 \mathrm{~kb}$, deletion carries the risk of removing (regulatory regions of) other genes. We therefore devised a strategy, whereby we first generated a CLIP-170 KO allele by inserting a GFPloxP-neo-loxP cassette in front of the first ATG codon. Subsequently, Cre-mediated excision of the neo-gene (via the loxP sites) generated the GFP-CLIP-170 KI allele.

The CLIP-170 KO allele turned out to be leaky in the adult lung and the embryo. It is possible that residual amounts of CLIP-170 are sufficient to carry KO mice through life. However, we emphasize that in the majority of the tissues investigated, including MEFs, we did not detect any CLIP-170, although there could exist small quantities of CLIP-170 that are below our levels of detection. Consistent with a mild phenotype in mice, cultured CLIP-170 KO MEFs do not show any obvious abnormalities under normal culture conditions. This is in line with RNAi studies that we performed in HeLa cells (Lansbergen et al. 2004). CLIP-115 might take over part of the function of CLIP-170 in cultured fibroblasts, since this protein can act as a MT rescue factor in $\mathrm{CHO}$ cells (Komarova et al. 2002). Our results in CLIP-170 KO MEFs do not concur with the mitotic phenotype of CLIP170 knock-down reported in HEp-2 cells (Wieland et al. 2004). One explanation, which may account for these apparently contradictory results, is that redundant pathways normally exist in mammalian mitosis, one of which requires CLIP-170. In HEp-2 cells, only this pathway would be operative and hence, knock-down of CLIP170 causes a severe defect. In cultured CLIP-170 KO MEFs (and perhaps in mice), redundant pathway(s) would still be functional and guide cells through mitosis. Interestingly, treatment of cultured ovarian cells with antisense oligonucleotides against restin revealed differential effects on cell proliferation in different cell types (Ho et al. 2003). More studies are required to address this issue.

The fact that male CLIP-170 KO mice are subfertile focused our attention on CLIP-170 function in male germ-cell development. We show that CLIP-170 is expressed in two waves during spermatogenesis, the first beginning in undifferentiated spermatogonia and lasting until the primary spermatocyte stage. Future studies will address possible role(s) of CLIP-170 at this stage, for ex- ample, in the maintenance of large syncytia capable of migration through the blood-testis barrier. The second wave of CLIP-170 expression occurs in spermiogenesis. We detected CLIP-170 very conspicuously on the spermatid manchette and the manchette ring. Consistent with this distribution, absence of CLIP-170 severely affects formation and/or maintenance of the spermatid manchette in KO mice.

The manchette consists of regularly spaced MTs that are, on one side, embedded in the manchette ring (Russell et al. 1991). It is likely that the MT-binding properties of CLIP-170 are relevant for its function in shaping the manchette. On the other hand, CLIP-170 interacts with the dynactin complex through $\mathrm{p} 150^{\text {Glued }}$ (Lansbergen et al. 2004). Arp1, which is another dynactin component, was shown to be associated with the fuzzy material that links manchette MTs to the nuclear envelope (Fouquet et al. 2000). A similar localization has been described for dynein (Yoshida et al. 1994). In CLIP-170 $\mathrm{KO}$ mice, dynactin distribution is perturbed. In EM micrographs from CLIP-170 KO mice, where we observed manchette MTs close to the nucleus, these MTs appeared to be linked to the nuclear envelope and the fuzzy material was present. Still, it could be that lack of CLIP170 mislocalizes the dynein-dynactin complex and that this is an underlying cause for the dissociation of manchette MTs from the nucleus.

Nuclear transformation into the characteristic hooklike shape depends on a functional manchette (Russell et al. 1991), and manchette abnormalities, such as those observed in the azh (abnormal spermatozoon head shape) mutant, correlate with sperm head deformities (Hugenholtz et al. 1984). The results in CLIP-170 KO mice, which produce sperm with abnormally shaped heads, confirm this conclusion. Interestingly, the azh mutation is due to a deletion in the Hook1 gene, which encodes a protein resembling CLIP-170: Hook1 is a dimer with an $\mathrm{N}$-terminal MT-binding domain, which is separated by a coiled-coil region from an organelle interaction domain (Mendoza-Lujambio et al. 2002).

CLIP-50 is a short testis-enriched C-terminal isoform of CLIP-170 (Tarsounas et al. 2001). Antisera raised against this protein should recognize CLIP-170, LORFCLIP-170, and CLIP-50 and cannot distinguish between these proteins in immunocytochemistry studies in wildtype mice (Tarsounas et al. 2001). We show that in our CLIP-170 KO mice, the expression of CLIP-50 is not affected. We observe weak staining with \#2360 antibodies in spermatids of these mice, while spermatids of wildtype mice are brightly stained by the same antibodies. These results indicate that CLIP-50 is a minor CLIP-170 isoform localized to spermatid manchettes. The functional relevance of CLIP-50 remains unclear.

Present and previous (Perez et al. 1999) results strongly suggest that GFP-CLIP-170 and CLIP-170 have very similar properties. In KI mice, GFP-CLIP-170 is expressed at similar levels compared with CLIP-170 in all tissues examined. Although tagged with GFP, the same isoforms are detected in homozygous KI mice as in wildtype animals. In addition, GFP-CLIP-170 can substitute 
for CLIP-170 in cultured cells, and the GFP-CLIP-170 KI mice are fertile, their spermatozoa have a normal hookshaped appearance, and, with the exception of crossbridge size and number, spermatid manchettes appear completely normal. In sections from KI testis, we detected GFP-CLIP-170 with anti-CLIP-170 and anti-LORF antisera, and the distribution of p150 Glued and EB3 was normal. Taken together, these data strongly suggest that GFP-CLIP-170 can functionally replace CLIP-170 in vivo in the testis. Thus, the dynamic behavior of GFP-CLIP170 in live testis tubules likely recapitulates that of CLIP-170.

Time-lapse imaging in testis tubules shows plus-endtracking GFP-CLIP-170 in spermatogonia. Bleaching studies in spermatogonia show that GFP-CLIP-170 is mobile, in line with the dynamic behavior of the protein. As the spermatogonia differentiate, GFP-CLIP-170 signal becomes initially more intense, and a gradual loss of plus-end-tracking GFP-CLIP-170 is observed. Bleaching studies confirm that GFP-CLIP-170 becomes less mobile. However, it is not completely immobile, and the protein even appears to pass from one cell of the syncytium to another.

In elongating spermatids, CLIP-170 is localized to the centrosomes and manchettes. The presence of $\gamma$-tubulin at the spermatid centrosome (Fouquet et al. 1998) suggests that the minus-ends of MTs may be nucleated at this location. Consequently, MT plus-ends could be located near the manchette ring, consistent with a prominent localization of +TIPs (CLIP-170, EB3, and dynactin) at this site. However, the manchette appears to be a stable structure; it lasts several days and is made of stabilized MTs, which contain detyrosinated $\alpha$-tubulin (Fouquet et al. 1997) and which are interconnected by cross-bridges. In agreement with manchette stability, both the time-lapse imaging and bleaching studies demonstrate that GFP-CLIP-170 is largely immobile in spermatids. It is therefore possible that CLIP-170 is involved in stabilization of manchette MTs.

Interestingly, high levels of overexpressed CLIP-170 induce the formation of stable MT bundles in cultured cells, with MT plus-ends assembled as patches in the cell periphery (e.g., Pierre et al. 1994). This was considered to be an artifact of overexpression, but it is noteworthy that relatively high levels of GFP-CLIP-170 in testis correlate with immobile protein and the presence of stable MTs. A structural, rather than a dynamic, function of CLIP-170 in spermatids correlates with the expression of a longer isoform, strongly extended in the coiled-coil region by inclusion of the LORF exon. LORF-encoded sequences could allow new protein-protein interactions of CLIP170. Alternatively, LORF could alter the folding properties of CLIP-170, thereby generating a protein with different MT-binding and/or other protein-binding characteristics.

Although manchettes are stable structures, injection of the MT-stabilizing agent taxol in rat testis disorganizes the MTs of the manchette (they are occasionally even enveloped by the nucleus) (Russell et al. 1991). Therefore, proper control of MT dynamics seems essen- tial for normal manchette formation and sperm head shaping. Our data emphasize a role for CLIP-170 in formation and/or maintenance of the spermatid manchette. This function may be more static and structural than would be expected on the basis of studies of the dynamics of CLIP-170 in cultured somatic cells. This might reflect an exceptional organization of MT dynamics during spermatogenesis, which, with the use of GFP-CLIP170 , has been visualized for the first time.

\section{Materials and methods}

Generation of CLIP-170 KO and GFP-CLIP-170 KI mice

Rat CLIP-170 cDNA was used to screen a mouse PAC library (RPCI-21), which was prepared from female 129S6/SvevTac mouse spleen genomic DNA (Osoegawa et al. 2000). A PAC clone, which hybridized with the $5^{\prime}$ part of the CLIP-170 cDNA, was used to isolate $\sim 7-\mathrm{kb} \mathrm{XbaI}$ and $\sim 6-\mathrm{kB}$ BamHI genomic DNA fragments, encompassing exons 2 and 3 of the CLIP-170 gene (Fig. 2A). These fragments were used to generate the targeting vector and the external probe (Fig. 2A), which were verified by sequencing. Targeting of E14 mouse embryonic stem (ES) cells, selection, and identification of the positive clones was performed as described previously (Hoogenraad et al. 2002). One targeted ES cell clone with the correct karyotype was chosen to convert the KO allele into a KI allele by Cre-mediated recombination in ES cells. Heterozygous CLIP-170 KO and KI ES cells were injected into C57Bl/6 blastocysts. Chimeric males were mated to $\mathrm{C} 57 \mathrm{Bl} / 6$ females to obtain germline transmission of the modified CLIP-170 alleles. Mouse genotyping was performed by PCR (Fig. 2B).

\section{Molecular biology and antibodies}

DNA, RNA, and protein isolations were performed according to standard procedures (Sambrook et al. 1989), with modifications (Hoogenraad et al. 2002). Southern, Northern, and Western blot analyses were performed as described (Hoogenraad et al. 2002). Multiple tissue Northern blots (mouse and human) were purchased from Clontech. RT-PCR analysis was performed according to the protocol of the manufacturer (Bio-Rad).

Antibodies against the CLIP-170 LORF exon (nucleotides 122672290-122670023 on mouse chromosome 5, version NCBI m32) were prepared as described (Hoogenraad et al. 2000), using a GST-fusion protein approach. We also used rabbit polyclonal antibodies \#2360 against CLIP-170 (Coquelle et al. 2002), \#2221 against CLIP-115 and CLIP-170, \#2238 against CLIP-115 (Hoogenraad et al. 2000), \#02-1005-07 against EB3 (Stepanova et al. 2003), and anti-GFP antibodies (Abcam). Mouse monoclonal antibodies were against $\beta$-tubulin (Sigma), p150 Glued and p50 (BD Biosciences), actin (Chemicon), and GFP (Santa Cruz, Roche). Secondary antisera were alkaline phosphatase-labeled anti-rabbit and anti-mouse antibodies (Sigma), FITC-labeled goat anti-rabbit antibody (Nordic Laboratories), and Alexa 594labeled anti-mouse antibody (Molecular Probes).

\section{Immunofluorescence and immunhistochemistry}

MEF, keratinocyte, and glial cell cultures were prepared as described (de Hoop et al. 1998; Hoogenraad et al. 2002). Fixation of cultured cells, incubation with antisera, and image acquisitions were performed as published (Akhmanova et al. 2001). For 3D 
representations, Z-stacks were imported into Volocity (Improvision), rendered, and exported as QuickTime movies.

For H/E staining, we fixed testis from adult mice in Bouin solution (overnight at $4^{\circ} \mathrm{C}$ ), washed and dehydrated material using ethanol and xylene, and embedded the testis in paraffin. Sections of $7 \mu \mathrm{m}$ were cut, mounted on SuperFrost Plus slides (Menzel-gläser), rehydrated, and stained with H/E.

For immunofluorescence analyses, testes were fixed in $4 \%$ paraformaldehyde in PBS overnight at $4^{\circ} \mathrm{C}$, and treated as above. Before immunostaining, sections were treated with the microwave (three times for $5 \mathrm{~min}, 750 \mathrm{~W}$ ) in $10 \mathrm{mM} \mathrm{NaCitrate} \mathrm{buffer}$ ( $\mathrm{pH}$ 6.0) to expose antigens and then stained using standard procedures. Alternatively, testis cryosections were fixed in $4 \%$ paraformaldehyde in PBS for $20 \mathrm{~min}$ and used directly for immunofluorescent staining.

\section{Analysis of mature sperm}

Animals were killed by cervical dislocation, and the epididymides were dissected. To obtain sperm for assessment of motility and morphology, the epididymis from one side was transferred into a Petri dish containing $0.5 \mathrm{~mL}$ of Dulbecco's medium (GIBCO) with $0.5 \%(\mathrm{w} / \mathrm{v})$ BSA. The cauda epididymis was cut, and sperm were allowed to swim out for $20 \mathrm{~min}$. The medium was carefully mixed, and aliquots were taken for analysis. Motility was evaluated by scoring the movement of 200 sperm in two different samples per mouse using phase contrast microscopy (400x magnification). Sperm morphology was assessed in smears stained with $\mathrm{H} / \mathrm{E}$ by viewing 200 sperm two times using phase-contrast microscopy (1000× magnification). The epididymis from the other side was used for sperm counting. The organ was transferred into a small conical glass grinder and homogenized by hand. The total number of sperm present in the epididymis was counted using a Neubauer haemocytometer and a phase contrast microscope (magnification 400×). At least 200 sperm in two different samples were counted.

\section{Time-lapse imaging and FRAP analysis}

For analysis of mitotic defects, dishes were transferred to a heated culture chamber $\left(37^{\circ} \mathrm{C}, 5 \% \mathrm{CO}_{2}\right)$ on a Zeiss Axiovert 200M microscope equipped with a 40× Plan-Neo DIC objective. We captured 12-bit phase contrast images using a Photometrics Coolsnap HQ charged-coupled device camera. Images were processed using MetaMorph software (Universal Imaging).

The fluorescence live imaging of cultured somatic cells was performed as described (Stepanova et al. 2003). For imaging and FRAP analysis of testis tubules, testes were decapsulated and the tissue was immersed in $20 \mathrm{~mL}$ of Dulbecco's phosphatebuffered saline (PBS) containing $0.9 \mathrm{mM} \mathrm{Ca}^{2+}, 0.5 \mathrm{mM} \mathrm{Mg}^{2+}, 8$ $\mathrm{mM}$ DL-lactic acid, and $5.6 \mathrm{mM}$ glucose $\left(\mathrm{PBS}^{+}\right)$, in the presence of collagenase ( $1 \mu \mathrm{g} / \mu \mathrm{L}$; Roche) and hyaluronidase $(0.5 \mu \mathrm{g} / \mu \mathrm{L}$; Roche) in a siliconated 100-mL Erlenmeyer flask. This flask was incubated in a $33^{\circ} \mathrm{C}$ waterbath, in air, and shaken at 90 cycles/ min for 15-20 min with an amplitude of $20 \mathrm{~mm}$. The incubation was terminated when the mild enzymatic digestion had resulted in dissociation of the interstitial tissue and disengagement of testis tubules. The tubules were separated from the interstitial cells by washing in $\mathrm{PBS}^{+}$twice. Next, several tubules were placed in a $30-\mathrm{mm}$ Petri dish, in $\mathrm{PBS}^{+}$supplemented with $0.2 \%$ bovine serum albumin (BSA). When required, $5 \mu \mathrm{g} / \mu \mathrm{L}$ Hoechst 33342 (Molecular Probes) was added. In this incubation system, the Hoechst stain is taken up only by nuclei on the basal side of the Sertoli cell barrier (also called "blood-testis barrier"). This allows for identification of all cells on the basal side of this barrier: peritubular cells, Sertoli cells, spermatogo- nia, and pre-leptotene spermatocytes. Selected tubules of $\sim 1 \mathrm{~cm}$ length were transferred into a drop of $30 \mu \mathrm{L}$ of $\mathrm{PBS}^{+}$with $0.2 \%$ BSA on a $24-\mathrm{mm}$ coverslip in a live cell chamber. A $16-\mathrm{mm}$ coverslip was placed on top, and this was overlaid with PBSsaturated mineral oil.

When applicable, the testis was injected through the rete testis with Hoechst 33342 and Trypan blue (Sigma) in 3-5 $\mu \mathrm{L}$ of PBS, $1 \mathrm{~h}$ prior to testis dissection, to allow spreading of the vital DNA stain throughout the adluminal compartment of the testis tubules and uptake by nuclei on the adluminal side of the Sertoli cell barrier. Trypan blue served as a marker for injected tubules. This method makes it possible to identify the germ cell types that have migrated through the Sertoli cell barrier.

The testis tubules were examined at $33^{\circ} \mathrm{C}$, using a Zeiss LSM510NLO confocal/multiphoton set up, to allow simultaneous acquisition of phase-contrast, GFP, and Hoechst images. GFP-CLIP-170 movement ex vivo was analyzed as published (Stepanova et al. 2003). FRAP analysis was carried out with the $63 \times$ planapochromat (1.4 NA) oil immersion lens. Bleaching of an outlined region of interest (ROI) was done with four iterations of the $488-\mathrm{nm}$ laser at full transmission $(2.6 \mathrm{~mW})$. Prior to and after bleaching, the laser was set at $2 \%$ transmission (55 $\mu \mathrm{W})$. The Zeiss LSM software was used to measure pixel intensities inside different ROIs. Recovery values were normalized to the prebleach values for each ROI. Values were imported into Aabel (Gigawiz) for graphical representation and statistical analysis.

\section{EM analysis}

After total body perfusion with $1.5 \%$ glutaraldehyde in $0.14 \mathrm{M}$ cacodylate buffer for $10 \mathrm{~min}$, testes were removed and transferred immediately into the same fixative. After $2 \mathrm{~h}$ of fixation at room temperature the testes were post-fixed in $1 \% \mathrm{OsO}_{4}$ for $1 \mathrm{~h}$ at $4^{\circ} \mathrm{C}$. The samples were dehydrated in a graded ethanol series and embedded in an epoxy resin. Ultrathin sections were stained with uranyl acetate and lead salts.

Quantification of cross-bridges was performed on electron micrographs with a final magnification of $160,000 \times$. The numbers of cross-sectioned MTs and cross-bridges were established by counting them on 10 electron micrographs of each condition (wild type, KI, and KO). Subsequently, the size of all crossbridges was measured. Statistical analysis was performed in Excel (Microsoft).

\section{Acknowledgments}

We thank Michiel de Ruyter for generating the anti-LORF antisera, Evelyn Wassenaar for dissection of testis tubules, Marja Ooms for help with immunohistochemistry, Jan de Wit for keratinocyte culture advice, An Langeveld for performing karyotype analysis, and Dick de Rooij (Utrecht University) for expert advice on male germ cell types. This work was supported by the Dutch Ministry of Economic Affairs (BSIK), the Netherlands Organization for Scientific Research (NWO Earth and Life Sciences and ZonMw), the Dutch Cancer Society, and an EEC integrated project on imaging.

\section{References}

Akhmanova, A. and Hoogenraad, C.C. 2005. Microtubule plusend-tracking proteins: Mechanisms and functions. Curr. Opin. Cell Biol. 17: 47-54.

Akhmanova, A., Hoogenraad, C.C., Drabek, K., Stepanova, T., Dortland, B., Verkerk, T., Vermeulen, W., Burgering, B.M., 
De Zeeuw, C.I., Grosveld, F., et al. 2001. Clasps are CLIP-115 and -170 associating proteins involved in the regional regulation of microtubule dynamics in motile fibroblasts. Cell 104: 923-935.

Arnal, I., Heichette, C., Diamantopoulos, G.S., and Chretien, D. 2004. CLIP-170/tubulin-curved oligomers coassemble at microtubule ends and promote rescues. Curr. Biol. 14: 20862095.

Bilbe, G., Delabie, J., Bruggen, J., Richener, H., Asselbergs, F.A., Cerletti, N., Sorg, C., Odink, K., Tarcsay, L., Wiesendanger, W., et al. 1992. Restin: A novel intermediate filament-associated protein highly expressed in the Reed-Sternberg cells of Hodgkin's disease. EMBO J. 11: 2103-2113.

Brunner, D. and Nurse, P. 2000. CLIP170-like tiplp spatially organizes microtubular dynamics in fission yeast. Cell 102: 695-704.

Busch, K.E., Hayles, J., Nurse, P., and Brunner, D. 2004. Tea2p kinesin is involved in spatial microtubule organization by transporting tiplp on microtubules. Dev. Cell 6: 831-843.

Carvalho, P., Gupta Jr., M.L., Hoyt, M.A., and Pellman, D. 2004. Cell cycle control of kinesin-mediated transport of Bik1 (CLIP-170) regulates microtubule stability and dynein activation. Dev. Cell 6: 815-829.

Coquelle, F.M., Caspi, M., Cordelieres, F.P., Dompierre, J.P., Dujardin, D.L., Koifman, C., Martin, P., Hoogenraad, C.C., Akhmanova, A., Galjart, N., et al. 2002. LIS1, CLIP-170's key to the dynein/dynactin pathway. Mol. Cell. Biol. 22: 3089-3102.

de Hoop, M.J., Meijn, L., and Dotti, C.G. 1998. Culturing hippocampal neurons and astrocytes from fetal rodent brain. In Cell biology: A laboratory handbook (ed. J.E. Celis), pp. 162163. Academic Press, San Diego.

De Zeeuw, C.I., Hoogenraad, C.C., Goedknegt, E., Hertzberg, E., Neubauer, A., Grosveld, F., and Galjart, N. 1997. CLIP-115, a novel brain-specific cytoplasmic linker protein, mediates the localization of dendritic lamellar bodies. Neuron 19: $1187-1199$.

Dujardin, D., Wacker, U.I., Moreau, A., Schroer, T.A., Rickard, J.E., and De Mey, J.R. 1998. Evidence for a role of CLIP-170 in the establishment of metaphase chromosome alignment. J. Cell Biol. 141: 849-862.

Fouquet, J.P., Kann, M.L., Pechart, I., and Prigent, Y. 1997. Expression of tubulin isoforms during the differentiation of mammalian spermatozoa. Tissue Cell 29: 573-583.

Fouquet, J.P., Kann, M.L., Combeau, C., and Melki, R. 1998. $\gamma$-Tubulin during the differentiation of spermatozoa in various mammals and man. Mol. Hum. Reprod. 4: 1122-1129.

Fouquet, J., Kann, M., Soues, S., and Melki, R. 2000. ARP1 in Golgi organisation and attachment of manchette microtubules to the nucleus during mammalian spermatogenesis. J. Cell Sci. 113: 877-886.

Fukata, M., Watanabe, T., Noritake, J., Nakagawa, M., Yamaga, M., Kuroda, S., Matsuura, Y., Iwamatsu, A., Perez, F., and Kaibuchi, K. 2002. Rac1 and Cdc42 capture microtubules through IQGAP1 and CLIP-170. Cell 109: 873-885.

Goodson, H.V., Skube, S.B., Stalder, R., Valetti, C., Kreis, T.E., Morrison, E.E., and Schroer, T.A. 2003. CLIP-170 interacts with dynactin complex and the APC-binding protein EB1 by different mechanisms. Cell Motil. Cytoskeleton 55: 156173.

Griparic, L. and Keller, T.C. 1998. Identification and expression of two novel CLIP-170/restin isoforms expressed predominantly in muscle. Biochim. Biophys. Acta 1405: 35-46.

Ho, S.M., Lau, K.M., Mok, S.C., and Syed, V.W. 2003. Profiling follicle stimulating hormone-induced gene expression changes in normal and malignant human ovarian surface epithelial cells. Oncogene 22: 4243-4256.

Hoogenraad, C.C., Akhmanova, A., Grosveld, F., De Zeeuw, C.I., and Galjart, N. 2000. Functional analysis of CLIP-115 and its binding to microtubules. J. Cell Sci. 113: 2285-2297.

Hoogenraad, C.C., Koekkoek, B., Akhmanova, A., Krugers, H., Dortland, B., Miedema, M., van Alphen, A., Kistler, W.M., Jaegle, M., Koutsourakis, M., et al. 2002. Targeted mutation of Cyln2 in the Williams syndrome critical region links CLIP-115 haploinsufficiency to neurodevelopmental abnormalities in mice. Nat. Genet. 32: 116-127.

Hugenholtz, A.P., Eng, V.W.S., and Bruce, W.R. 1984. Mouse News Lett. 71: 34-35.

Kierszenbaum, A.L. 2001. Spermatid manchette: Plugging proteins to zero into the sperm tail. Mol. Reprod. Dev. 59: $347-$ 349.

Komarova, Y.A., Akhmanova, A.S., Kojima, S., Galjart, N., and Borisy, G.G. 2002. Cytoplasmic linker proteins promote microtubule rescue in vivo. J. Cell Biol. 159: 589-599.

Lansbergen, G., Komarova, Y., Modesti, M., Wyman, C., Hoogenraad, C.C., Goodson, H.V., Lemaitre, R.P., Drechsel, D.N., van Munster, E., Gadella Jr., T.W., et al. 2004. Conformational changes in CLIP-170 regulate its binding to microtubules and dynactin localization. I. Cell Biol. 166: 10031014.

Lantz, V.A. and Miller, K.G. 1998. A class VI unconventional myosin is associated with a homologue of a microtubulebinding protein, cytoplasmic linker protein-170, in neurons and at the posterior pole of Drosophila embryos. J. Cell Biol. 140: 897-910.

Mendoza-Lujambio, I., Burfeind, P., Dixkens, C., Meinhardt, A., Hoyer-Fender, S., Engel, W., and Neesen, J. 2002. The Hook1 gene is non-functional in the abnormal spermatozoon head shape (azh) mutant mouse. Hum. Mol. Genet. 11: 16471658.

Osoegawa, K., Tateno, M., Woon, P.Y., Frengen, E., Mammoser, A.G., Catanese, J.J., Hayashizaki, Y., and de Jong, P.J. 2000. Bacterial artificial chromosome libraries for mouse sequencing and functional analysis. Genome Res. 10: 116-128.

Perez, F., Diamantopoulos, G.S., Stalder, R., and Kreis, T.E. 1999. CLIP-170 highlights growing microtubule ends in vivo. Cell 96: 517-527.

Pierre, P., Scheel, J., Rickard, J.E., and Kreis, T.E. 1992. CLIP-170 links endocytic vesicles to microtubules. Cell 70: 887-900.

Pierre, P., Pepperkok, R., and Kreis, T.E. 1994. Molecular characterization of two functional domains of CLIP-170 in vivo. J. Cell Sci. 107: 1909-1920.

Rickard, J.E. and Kreis, T.E. 1990. Identification of a novel nucleotide-sensitive microtubule-binding protein in HeLa cells. J. Cell Biol. 110: 1623-1633.

Russell, L.D., Russell, J.A., MacGregor, G.R., and Meistrich, M.L. 1991. Linkage of manchette microtubules to the nuclear envelope and observations of the role of the manchette in nuclear shaping during spermiogenesis in rodents. Am. J. Anat. 192: 97-120.

Sambrook, J., Fritsch, E.F., and Maniatis, T. 1989. Molecular cloning: A laboratory manual, 2nd ed. Cold Spring Harbor Laboratory Press, Cold Spring Harbor, NY.

Schuyler, S.C. and Pellman, D. 2001. Microtubule 'plus-endtracking proteins': The end is just the beginning. Cell 105: 421-424.

Stepanova, T., Slemmer, J., Hoogenraad, C.C., Lansbergen, G., Dortland, B., De Zeeuw, C.I., Grosveld, F., van Cappellen, G., Akhmanova, A., and Galjart, N. 2003. Visualization of microtubule growth in cultured neurons via the use of EB3- 
GFP (end-binding protein 3-green fluorescent protein). I. Neurosci. 23: 2655-2664.

Tai, C.Y., Dujardin, D.L., Faulkner, N.E., and Vallee, R.B. 2002. Role of dynein, dynactin, and CLIP-170 interactions in LIS1 kinetochore function. J. Cell Biol. 156: 959-968.

Tarsounas, M., Pearlman, R.E., and Moens, P.B. 2001. CLIP-50 immunolocalization during mouse spermiogenesis suggests a role in shaping the sperm nucleus. Dev. Biol. 236: 400-410.

Vigodner, M., Lewin, L.M., Glaser, T., Shochat, L., Mittelman, L., and Golan, R. 2002. Use of confocal microscopy for the study of spermatogenesis. Methods Cell Sci. 24: 169-180.

Wieland, G., Orthaus, S., Ohndorf, S., Diekmann, S., and Hemmerich, P. 2004. Functional complementation of human centromere protein A (CENP-A) by Cse4p from Saccharomyces cerevisiae. Mol. Cell. Biol. 24: 6620-6630.

Wolosewick, J.J. and Bryan, J.H. 1977. Ultrastructural characterization of the manchette microtubules in the seminiferous epithelium of the mouse. Am. J. Anat. 150: 301-331.

Yoshida, T., Ioshii, S.O., Imanaka-Yoshida, K., and Izutsu, K. 1994. Association of cytoplasmic dynein with manchette microtubules and spermatid nuclear envelope during spermiogenesis in rats. J. Cell Sci. 107: 625-633. 


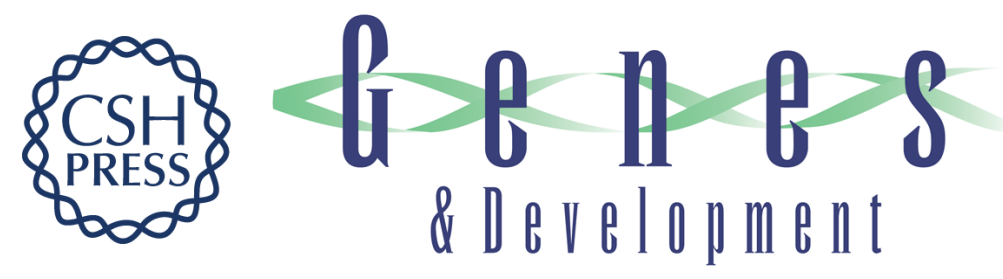

\section{The microtubule plus-end-tracking protein CLIP-170 associates with the spermatid manchette and is essential for spermatogenesis}

Anna Akhmanova, Anne-Laure Mausset-Bonnefont, Wiggert van Cappellen, et al.

Genes Dev. 2005, 19:

Access the most recent version at doi:10.1101/gad.344505

Supplemental http://genesdev.cshlp.org/content/suppl/2005/09/29/19.20.2501.DC1
Material

References This article cites 39 articles, 14 of which can be accessed free at:

http://genesdev.cshlp.org/content/19/20/2501.full.html\#ref-list-1

License

Email Alerting

Receive free email alerts when new articles cite this article - sign up in the box at the top

Service

right corner of the article or click here.

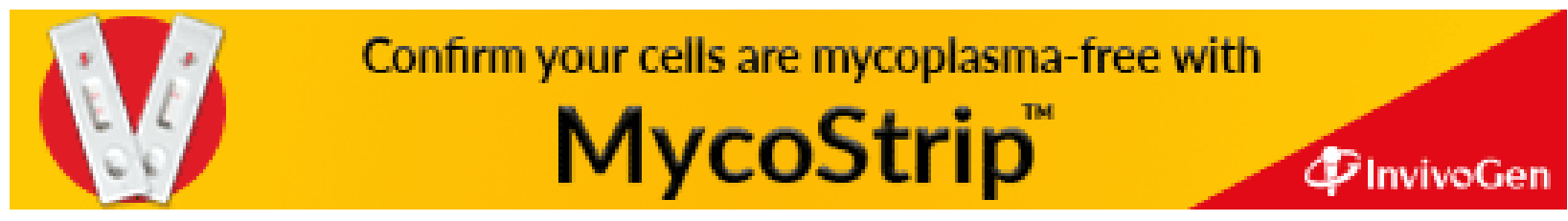

